\title{
The effect of telemedicine in critically ill patients: systematic review and meta-analysis
}

\author{
M Elizabeth Wilcox ${ }^{1 *}$ and Neill KJ Adhikari ${ }^{2}$
}

\begin{abstract}
Introduction: Telemedicine extends intensivists' reach to critically ill patients cared for by other physicians. Our objective was to evaluate the impact of telemedicine on patients' outcomes.

Methods: We searched electronic databases through April 2012, bibliographies of included trials, and indexes and conference proceedings in two journals (2001 to 2012). We selected controlled trials or observational studies of critically ill adults or children, examining the effects of telemedicine on mortality. Two authors independently selected studies and extracted data on outcomes (mortality and length of stay in the intensive care unit (ICU) and hospital) and methodologic quality. We used random-effects meta-analytic models unadjusted for case mix or cluster effects and quantified between-study heterogeneity by using $P^{2}$ (the percentage of total variability across studies attributable to heterogeneity rather than to chance).

Results: Of 865 citations, 11 observational studies met selection criteria. Overall quality was moderate (mean score on Newcastle-Ottawa scale, 5.1/9; range, 3 to 9). Meta-analyses showed that telemedicine, compared with standard care, is associated with lower ICU mortality (risk ratio (RR) 0.79; $95 \%$ confidence interval (Cl), 0.65 to 0.96; nine studies, $n=23,526$; $P^{2}=70 \%$ ) and hospital mortality (RR, $0.83 ; 95 \% \mathrm{Cl}, 0.73$ to 0.94 ; nine studies, $n=47,943 ; P^{2}=72 \%$ ). Interventions with continuous patient-data monitoring, with or without alerts, reduced ICU mortality (RR, $0.78 ; 95 \% \mathrm{Cl}, 0.64$ to 0.95 ; six studies, $n=21,384 ; P^{2}=74 \%$ ) versus those with remote intensivist consultation only (RR, $0.64 ; 95 \% \mathrm{Cl}, 0.20$ to 2.07 ; three studies, $n=2,142 ; P=71 \%$ ), but effects were statistically similar (interaction $P=0.74$ ). Effects were also similar in higher ( $R R, 0.83 ; 95 \% \mathrm{Cl}, 0.68$ to 1.02 ) versus lower (RR, $0.69 ; 95 \% \mathrm{Cl}, 0.40$ to 1.19 ; interaction, $P=0.53$ ) quality studies. Reductions in ICU and hospital length of stay were statistically significant (weighted mean difference (telemedicine-control), -0.62 days; $95 \% \mathrm{Cl},-1.21$ to -0.04 days and -1.26 days; $95 \% \mathrm{Cl},-2.49$ to -0.03 days, respectively; $P^{2}>90 \%$ for both).
\end{abstract}

Conclusions: Telemedicine was associated with lower ICU and hospital mortality among critically ill patients, although effects varied among studies and may be overestimated in nonrandomized designs. The optimal telemedicine technology configuration and dose tailored to ICU organization and case mix remain unclear.

\section{Introduction}

High-intensity physician staffing in intensive care units (ICUs), known as a closed ICU model, is defined as mandatory transfer of responsibility for the care of every critically ill patient to an intensivist-led team or mandatory consultation by an intensivist [1]. This model of staffing is associated with an increased use of evidence-based treatments [2] and significant reductions in mortality and length of stay (LOS) [3,4]. However, projected population

\footnotetext{
* Correspondence: elizabeth.wilcox@utoronto.ca

'Department of Medicine, Toronto Western Hospital, and University of Toronto, McLaughlin Wing 2-411H, 399 Bathurst Street, Toronto ON M5T 2S8, Canada

Full list of author information is available at the end of the article
}

demands for intensive care will exceed the number of available intensivists, implying that many critically ill patients will be cared for in low-intensity staffing ICUs (an open model), in which any physician can admit and care for patients without the involvement of an intensivist [5].

Telemedicine, broadly defined as the exchange of medical information via electronic communication, may help to fill gaps in intensivist coverage and give all patients access to specialty care 24 hours per day, 7 days per week [6]. It allows real-time exchange of clinical data and direct interaction among critical care providers across long distances and provides decision support to underserviced rural areas, small hospitals without access to intensivists [7], and large hospitals with low-intensity
C Biomed Central

() 2012 Wilcox et al.; licensee BioMed Central Ltd. This is an open access article distributed under the terms of the Creative Commons Attribution License (http://creativecommons.org/licenses/by/2.0), which permits unrestricted use, distribution, and reproduction in any medium, provided the original work is properly cited. 
physician-staffing models or nocturnal physician shortages. Some applications also contain decision-support tools to facilitate implementation of best practices and alarms to alert providers to sudden changes in patient status [8-12].

A recent systematic review found that telemedicine, as compared with standard of care, decreased mortality in patients admitted to ICUs [13]. However, it remains unclear whether an active telemedicine system (for example, one with continuous monitoring of patient data with computer-generated alerts) is required for beneficial clinical effects or whether more-passive systems (such as remote intensivist consultation alone) would suffice. The objective of our systematic review was to determine the effect of telemedicine on ICU mortality in critically ill patients, focusing on subgroup effects related to the intensity of the intervention and quality of the study.

\section{Materials and methods}

\section{Literature search}

OVID versions of MEDLINE (1948 to April, Week 2, 2012), EMBASE Classic and EMBASE (1947 to week 16, 2012); Web of Science (1970 through to April, Week 2, 2012); and the Cochrane Central Register of Controlled Trials (first quarter, 2012) were searched (see Appendix A in Additional file 1). All literature searches were conducted with the aid of an experienced information specialist. We also handsearched two major intensive care journals, Critical Care Medicine and Intensive Care Medicine (2001 to 2012); conference abstracts from annual meetings of the Society of Critical Care Medicine and the European Society of Intensive Care Medicine (2001 to 2012); and bibliographies of included studies and personal files. No language restrictions were imposed. Two reviewers independently reviewed all citations; the full text of any citation considered potentially relevant by any reviewer was retrieved. The degree of interrater agreement for study selection was determined by using kappa, with standard definitions for poor $(<0.20)$, fair $(0.21$ to 0.40$)$, moderate ( 0.41 to 0.60$)$, good (0.61 to 0.80$)$, and very good ( 0.81 to $1.00)$ agreement [14].

\section{Study selection}

Two unblinded reviewers assessed full-text reports and included studies meeting the following criteria: (a) design: randomized and quasi-randomized (allocation by hospital file number, for example) controlled trials or observational studies; (b) population: patients admitted to an ICU; (c) intervention: telemedicine compared with standard of care; and (d) outcome reported: ICU or hospital mortality.

Studies also were considered for inclusion if the telemedicine intervention included cointerventions (for example, computerized physician order entry) and regardless of the degree of exposure to the telemedicine intervention (for example, 24 hours per day, 7 days per week versus nighttime coverage only).

When authors reported data in several publications that included the same patient population, only the most recent or complete study was included in the analysis. Authors were contacted in an attempt to clarify methodology and to request additional data when a study was excluded because the data could not be used [15-21].

\section{Data abstraction and validity assessment}

Two reviewers (MEW, NA) abstracted data including patient population, telecommunication methods, and patient outcomes from included studies. We classified the telemedicine intervention as active (continuous patient data monitoring with computer-generated alerts), high-intensity passive (continuous patient data monitoring without computer-generated alerts), or low-intensity passive (no continuous data monitoring). Study quality was assessed by using the Newcastle-Ottawa score for cohort studies [22].

\section{Data analysis}

The primary outcome of this systematic review was ICU mortality. Secondary outcomes included hospital mortality and ICU and hospital length of stay.

Review Manager 5.0.22 (The Cochrane Collaboration, Oxford, England) was used to calculate pooled risk ratios (RRs) for dichotomous outcomes and pooled weighted mean differences (WMDs) for continuous outcomes, both with 95\% confidence intervals (CIs) were calculated. Random-effects models, which incorporate between-trial heterogeneity and thus generally give wider confidence intervals when heterogeneity is present, were used. Because of variability in methods and reporting of adjustment for case-mix and cluster effects among included studies, meta-analyses used unadjusted data. We assessed heterogeneity among trials by using $I^{2}$, the percentage of total variability across studies attributable to heterogeneity rather than to chance $[23,24]$ and used published guidelines for low $\left(I^{2}=25 \%\right.$ to $\left.49 \%\right)$, moderate $\left(I^{2}=50 \%\right.$ to $74 \%)$, and high heterogeneity $\left(I^{2} \geq 75 \%\right)$ [23]. For the primary outcome of ICU mortality, we inspected a funnel plot (scatterplot of standard error of $\log R R$ against RR for each study) and used the Peters regression test [25] to assess for the presence of publication bias. Continuous variables are expressed as mean (standard deviation, SD), unless otherwise indicated.

Subgroup analyses were performed for ICU mortality stratified by (a) study quality (higher, defined as Newcastle-Ottawa score $\geq 6$, versus lower), and (b) type of telemedicine intervention: active or high-intensity passive systems versus low-intensity passive systems. In a post 
hoc modification of the second analysis, we compared active with low-intensity passive systems. To test for a subgroup effect, pooled risk ratios (RRs) for each subgroup were compared by using a $z$ test [26].

\section{Results}

\section{Study flow}

The search strategy yielded 865 citations (see Figure 1). Seventy-three articles were retrieved for detailed evaluation, of which 62 were excluded (see Appendix B in Additional file 1). Eleven studies $(n=49,457)$ met criteria for inclusion [8-12,27-32]. No authors successfully contacted $[16,17,20,21]$ were able to provide additional data.

\section{Description of included studies}

We did not find any randomized or quasi-randomized trials of telemedicine. Eleven studies used a before-after observational design [8-12,27-32] (Table 1), of which one used a prospective stepped-wedge design [29]. Reviewers achieved very good agreement on study inclusion (kappa 0.92; 95\% CI, 0.83 to 1.00). Studies enrolled a median of 2,027 patients (range, 429 to 24,656 ) and were conducted in one $[8,10,11,27-31]$ or two [12] hospitals, except for two studies that each implemented telemedicine across five hospitals $[9,32]$. All studies were conducted in the United States. Two studies excluded patients in the ICU for less than 4 hours or transferred from another facility $[11,12]$. One study included only patients with neurologic diagnoses (stroke, intracranial hemorrhage, traumatic brain injury) [28], another study specifically excluded the neurotrauma ICU from the telemedicine intervention [9], and a third study restricted the intervention to patients with a medical diagnosis [11]. The median study duration in the intervention groups was 37 weeks (range, 10 to 144 weeks) [8-12,27-32].

The median of the mean ages of patients was 62 years (range, 5.5 to 66 years) and 64 years (range, 5.3 to 65 years) in the standard-care and intervention groups, respectively. On average, $54 \%$ of patients were men (range, 51\% to 57\%) in the six studies providing this information [8,10-12,29]. Patients had high illness severity, measured by using a variety of scoring systems (Table 1).

\section{Details of the interventions}

Three studies implemented a low-intensity passive system $[10,27,28]$ (Table 2). One study implemented a lowintensity passive system with video teleconferencing equipment exclusively for clinical consultation [27]. On average, 1.5 consults occurred per patient (range, one to

865 citations initially identified from electronic databases

864 From Medline, Web of Science, EMBASE, EMBASE Classic

1 From Cochrane Central Register of Controlled Trials
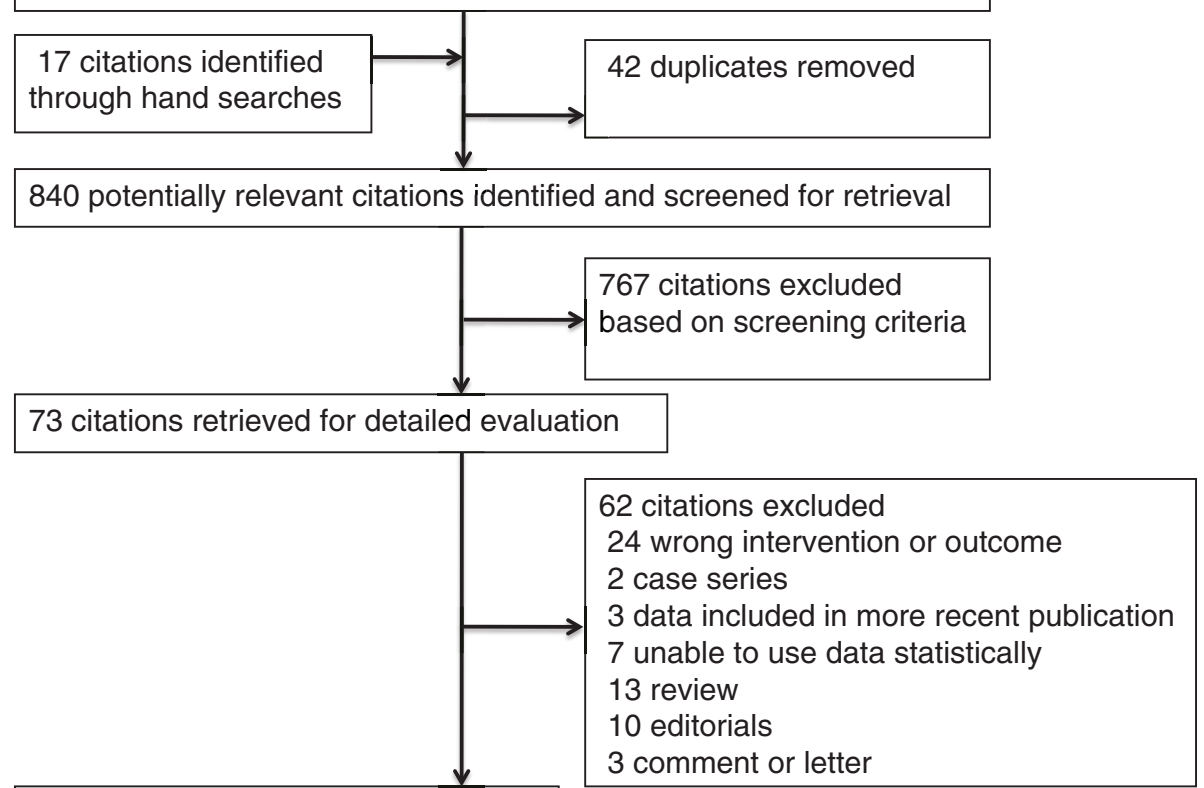

11 studies included in meta-analysis

Figure 1 Flowchart of study selection for the systematic review. 
Table 1 Characteristics of the included studies

\begin{tabular}{|c|c|c|c|c|c|c|c|c|}
\hline \multirow[t]{2}{*}{ Source } & \multirow{2}{*}{$\begin{array}{l}\text { ICUs/ } \\
\text { hospitals, } n\end{array}$} & \multirow{2}{*}{$\begin{array}{l}\text { Patients }^{\text {a }} \text { (total/ } \\
\text { pre/post), } n\end{array}$} & \multicolumn{2}{|c|}{ Age (years) } & \multicolumn{2}{|c|}{ Sex (\% male) } & \multicolumn{2}{|c|}{ Illness severity } \\
\hline & & & Control & Telemedicine & Control & Telemedicine & Control & Telemedicine \\
\hline Rosenfeld et al. 2000 [10] & $1 / 1$ & $628 / 227 / 201$ & 61 & 61 & 56 & 57 & $\begin{array}{l}\text { APACHE III } \\
37\end{array}$ & APACHE III 38 \\
\hline Breslow et al. 2004 [8] & $2 / 1$ & $2,140 / 1,396 / 744$ & 61 & 60 & 56 & 50 & $\begin{array}{l}\text { APACHE III } \\
\text { APS } 39\end{array}$ & $\begin{array}{l}\text { APACHE III } \\
\text { APS } 38\end{array}$ \\
\hline Marcin et al. 2004 [27] & $1 / 1$ & $296 / 249 / 47$ & 5.5 & 5.3 & NR & NR & $\begin{array}{l}\text { PRISM III } \\
7.5\end{array}$ & $\begin{array}{l}\text { PRISM III } \\
9.6\end{array}$ \\
\hline Kohl et al. 2007 [30] & $1 / 1$ & $2,811 / 189 / 2,622$ & NR & NR & NR & NR & NR & NR \\
\hline Vespa et al. 2007 [28] & $1 / 1$ & $1,218 / 578 / 640$ & NR & NR & NR & NR & NR & NR \\
\hline Norman et al. 2009 [31] & $1 / 1$ & $1,275 / 356 / 919$ & NR & NR & NR & NR & $\begin{array}{l}\text { APACHE IV } \\
57\end{array}$ & APACHE IV 53 \\
\hline Thomas et al. 2009 [9] & $6 / 5$ & $4,142 / 2,034 / 2,108$ & 60 & 59 & 51 & 53 & SAPS $\| 35$ & SAPS II 34 \\
\hline McCambridge et al. 2010 [11] & $3 / 1$ & $1,913 / 954 / 959$ & 65 & 64 & 50 & 50 & $\begin{array}{l}\text { APACHE IV } \\
\text { APS } 57\end{array}$ & $\begin{array}{l}\text { APACHE IV } \\
\text { APS } 58\end{array}$ \\
\hline Morrison et al. 2010 [12] & $4 / 2$ & $4,088 / 1,371 / 2,717$ & 64 & 65 & 56 & 52 & $\begin{array}{l}\text { APACHE III } \\
49\end{array}$ & APACHE III 48 \\
\hline Lilly et al. 2011 [29] & $7 / 1$ & $6,290 / 1,529 / 4,761$ & 62 & 64 & 57 & 57 & $\begin{array}{l}\text { APACHE III } \\
45\end{array}$ & APACHE III 58 \\
\hline Willmitch et al. 2012 [32] & $10 / 5$ & $24,656 / 6,504 / 18,152$ & NR & NR & NR & NR & CMI 2.68 & CMI 2.77 \\
\hline
\end{tabular}

All studies were conducted in the United States. Of the 49,457 patients enrolled, 33,870 were enrolled in the telemedicine arm. Continuous data are expressed as mean (SD). ${ }^{a}$ The number of patients in each category corresponds to the number used in the meta-analysis. APACHE, Acute Physiology and Chronic Health Evaluation; APS, Acute Physiology Score; CMI, Case Mix Index; ICU, intensive care unit; ISS, Injury Severity Score; NR, not reported; PRISM, Pediatric Risk of Mortality Score; SAPS, Simplified Acute Physiology Score.

seven) during the ICU stay [27]. Another study used a low-intensity passive system featuring a robotic telepresence program and a clinical information system as a cointervention [28]. In this study, the tele-ICU physician assessed patients twice daily and reviewed each patient during nighttime rounds (minimum of 5 minutes per patient; mean, 52 minutes per night). The third study used video-conferencing equipment to perform formal rounds with either the attending physician or senior housestaff [10]. Tele-ICU physicians devoted 4 to 5 hours per day to patient care; physiological data from patients were stored and viewed every 2 hours.

Five studies examined active interventions with audiovideo monitoring and a data-monitoring system that generated alerts based on abnormal vital signs or laboratory or radiologic tests $[9,11,12,29,32]$, and two studies did not provide enough detail to classify them as either highintensity passive or active systems $[8,30,31]$. Six studies used VISICU software (eICU program of intensivist-led remote monitoring; VISICU Inc., Baltimore, MD, USA, and Amsterdam, The Netherlands) [8,9,12,29,31,32]. Patients were monitored continuously for 12 to 24 hours per day, with tele-ICU physicians rounding on all monitored patients every 1 to 4 hours. Studies provided varying levels of detail on the dose of the intervention; three studies reported on actual patient-care time [10,28] or number of consultations provided [27], two studies described the number and nature of patient-care orders given by the tele-ICU $[9,29]$, and two studies described different levels of tele-ICU involvement in patient care, depending on hospital physician preference in primarily open ICUs $[12,32]$. One study measured changes in adoption of best practices with telemedicine [29], whereas another reported that the tele-ICU had the authority to make recommendations regarding best practices for some patients, but did not report process-of-care data [32].

\section{Study quality}

Overall study quality was moderate (mean score on Newcastle-Ottawa scale, 5.1; range, 3 to 9 (maximum possible score); see Appendix C in Additional file 1). A minority of studies adequately reported details of the uptake of the intervention $[9,10,27-29]$ and assessment of outcome $[10,29]$.

\section{Primary and secondary outcomes}

Pooled data from nine studies (23,526 patients, 14,799 in the telemedicine group) showed that telemedicine reduced ICU mortality (RR, 0.79 ; $95 \%$ CI, 0.65 to $0.96 ; P=0.02$; Figure 2, upper panel). Visual inspection of a funnel plot and Peters regression test $(P=0.45)$ did not suggest publication bias (see Appendix D in Additional file 1). Similarly, data from nine studies (47,943 patients, 33,183 in the telemedicine group) showed that telemedicine reduced hospital mortality (RR, $0.83 ; 95 \% \mathrm{CI}, 0.73$ to $0.94 ; P=0.004$; 
Table 2 Telemedicine intervention in the included studies

\begin{tabular}{|c|c|c|c|c|}
\hline $\begin{array}{l}\text { Source } \\
\text { Study periods }\end{array}$ & $\begin{array}{l}\text { Type of hospital/ICU } \\
\text { ICU staffing model } \\
\text { Type of intervention }\end{array}$ & Intervention details & Intervention dose & $\begin{array}{l}\text { Equipment } \\
\text { cointerventions }\end{array}$ \\
\hline $\begin{array}{l}\text { Rosenfeld et al. } \\
\text { 2000 }[10] \\
\text { Pre 1: } 1 \text { Sept-18 } \\
\text { Dec } 1996 \\
\text { Pre 2: } 1 \text { Feb-18 } \\
\text { May } 1997^{\mathrm{b}} \\
\text { Post: } 1 \text { Sept-18 } \\
\text { Dec } 1997\end{array}$ & $\begin{array}{l}\text { Academic-affiliated community hospital; } \\
\text { surgical ICU } \\
\text { Open model } \\
\text { Low-intensity passive }\end{array}$ & $\begin{array}{l}\text { Tele-intensivist interacted with patients and } \\
\text { healthcare personnel via dedicated video } \\
\text { conferencing and data transmission } \\
\text { equipment } 24 \text { hours/day } \\
\text { Clinical and stored physiologic data } \\
\text { reviewed q2hours }\end{array}$ & $\begin{array}{l}\text { Formal video conferencing rounds occurred } \\
\text { on } 50 \% \text { of days; otherwise, intensivist } \\
\text { discussed each case with senior housestaff } \\
\text { or attending physician } \\
\text { Tele-intensivists spent } 4 \text { to } 5 \text { hours/day on } \\
\text { clinical care }\end{array}$ & $\begin{array}{l}\text { Spacelabs Medical, Seattle WA } \\
\text { None }\end{array}$ \\
\hline $\begin{array}{l}\text { Breslow et al. } 2004 \\
\text { [8] } \\
\text { Pre: } 1 \text { July } 1999-20 \\
\text { June } 2001 \\
\text { Post: } 1 \text { Jan-30 } \\
\text { June } 2001\end{array}$ & $\begin{array}{l}\text { Tertiary care, teaching; medical and surgical } \\
\text { ICUs } \\
\text { Closed unit for teaching team of medical } \\
\text { ICU patients ( } 40 \%) \text {; open model for } \\
\text { remaining medical ICU patients and } \\
\text { surgical ICUC } \\
\text { High-intensity passive or active (alerts not } \\
\text { clearly described) }\end{array}$ & $\begin{array}{l}\text { Tele-ICU staff (board certified intensivist, } \\
\text { nurse) monitored all patients } 19 \text { hours/day } \\
\text { (1200-0700) } \\
\text { Admitting physician determined tele-ICU } \\
\text { decision-making authority (all versus some } \\
\text { versus off-hours) } \\
\text { Tele-ICU reviewed patient data q4hours }\end{array}$ & Not described & $\begin{array}{l}\text { VISICU Inc. (elCU CARE), Baltimore MD } \\
\text { None }\end{array}$ \\
\hline $\begin{array}{l}\text { Marcin et al. } 2004 \\
\text { [27] } \\
\text { Pre: Oct 1997-Sept } \\
\text { 1998 } \\
\text { Post: Apr 2000-Apr } \\
\text { 2002 }\end{array}$ & $\begin{array}{l}\text { Tertiary referral; adult ICU (with some } \\
\text { pediatric patients) } \\
\text { Pediatric intensivist during baseline period } \\
\text { only } \\
\text { Low-intensity passive }\end{array}$ & $\begin{array}{l}\text { Consultation (at discretion of admitting } \\
\text { physician) with tele-pediatric intensivist } \\
\text { using portable telemedicine unit in pediatric } \\
\text { ICU and five consultants' homes available } 24 \\
\text { hours/day within } 15 \text { minutes }\end{array}$ & $\begin{array}{l}\text { Number of consultations, one to seven per } \\
\text { patient (median, } 1 ; \text { mean, } 1.5 \text { ) }\end{array}$ & $\begin{array}{l}\text { Tandberg } 800 \text { video conference units } \\
\text { None }\end{array}$ \\
\hline $\begin{array}{l}\text { Kohl et al. } 2007 \\
\text { [30] } \\
\text { Dates not } \\
\text { reported }\end{array}$ & $\begin{array}{l}\text { Academic; surgical ICU } \\
\text { Staffing model not described } \\
\text { High-intensity passive or active (based on } \\
\text { vendor) }\end{array}$ & $\begin{array}{l}\text { Tele-ICU staffed by board certified } \\
\text { intensivists; no further details provided }\end{array}$ & Not described & $\begin{array}{l}\text { VISICU Inc. (elCU CARE), Baltimore MD } \\
\text { None }\end{array}$ \\
\hline $\begin{array}{l}\text { Vespa et al. } 2007 \\
\text { [28] } \\
\text { Pre: 2003-2004 } \\
\text { fiscal year } \\
\text { Post: June 2005- } \\
\text { June } 2006\end{array}$ & $\begin{array}{l}\text { Academic; neurologic ICU } \\
\text { Staffing model not described; tele- } \\
\text { intensivist same as on-site intensivist } \\
\text { Low-intensity passive }\end{array}$ & $\begin{array}{l}\text { Robotic telepresence program for live } \\
\text { interactive consultation and review of } \\
\text { physiologic trends with intensivist [2000- } \\
0000 \text { (weekdays); } 1800 \text { (weekends)] } \\
\text { Each patient reviewed for } \geq 5 \text { minutes }\end{array}$ & $\begin{array}{l}\text { Mean, two sessions/day } \\
\text { Mean night-time rounding session, } 52 \\
\text { minutes }\end{array}$ & $\begin{array}{l}\text { Robot: InTouch Health, Santa Barbara } \\
\text { CA } \\
\text { Informatics system: Global Care Quest, } \\
\text { Aliso Viejo CA } \\
\text { Integrated clinical information system } \\
\text { Paging protocol with goal of attending } \\
\text { physician response within } 15 \text { minutes } \\
\end{array}$ \\
\hline $\begin{array}{l}\text { Norman et al. } \\
2009 \text { [31] } \\
\text { Pre: Jan-Mar } 2008 \\
\text { Post: Jan-Mar and } \\
\text { Apr-June 2009 }\end{array}$ & $\begin{array}{l}\text { Hospital not described; medical-surgical } \\
\text { ICU } \\
\text { Staffing model not described } \\
\text { High-intensity passive or active (alerts not } \\
\text { clearly described) }\end{array}$ & $\begin{array}{l}\text { Tele-ICU staff ("team" included nurse; } \\
\text { intensivist presence not specifically stated) } \\
\text { reviewed patients; no further details } \\
\text { provided }\end{array}$ & Not described & $\begin{array}{l}\text { VISICU Inc. (elCU CARE), Baltimore MD } \\
\text { Electronic discharge management tool }\end{array}$ \\
\hline $\begin{array}{l}\text { Thomas et al. } \\
2009 \text { [9] } \\
\text { Pre: Jan 2003-Aug } \\
2005 \\
\text { Post (staggered } \\
\text { roll-out): July } \\
\text { 2004-July } 2006\end{array}$ & $\begin{array}{l}\text { Closed }{ }^{f} \text { medical and trauma/surgical ICU in } \\
\text { tertiary care teaching hospital; two open } \\
\text { medical-surgical ICUs in two small } \\
\text { community hospitals; two open medical- } \\
\text { surgical ICUs in two large urban hospitals } \\
\text { Active }\end{array}$ & $\begin{array}{l}\text { Tele-ICU staffed by two physicians (noon -7 } \\
\text { am Monday-Friday, } 24 \text { hours/day weekends), } \\
\text { four registered nurses, and two } \\
\text { administrative technicians } \\
\text { Rounds frequency: severely ill q1 hour, } \\
\text { moderately ill q2 hours, relatively stable q4 } \\
\text { hours } \\
\text { Local physicians delegated to tele-ICU } \\
\text { authority for full treatment ( } 31 \% \text { of patients) } \\
\text { or for intervention only for life-threatening } \\
\text { events (66\%) }\end{array}$ & $\begin{array}{l}\text { Tele-ICU physicians gave 1,446 orders in } 60 \\
\text { days (four ICUs) } \\
\text { Two closed ICUs, } 5.3 \text { orders/day ( } 7 \% \text { high- } \\
\text { level interventions, (for example, code } \\
\text { supervision, ventilator management) } \\
\text { Two open ICUs, } 18.5 \text { orders/day ( } 26 \% \text { high- } \\
\text { level) }\end{array}$ & $\begin{array}{l}\text { VISICU Inc. (eICU CARE), Baltimore MD } \\
\text { None }\end{array}$ \\
\hline
\end{tabular}


Table 2 Telemedicine intervention in the included studies (Continued)

\begin{tabular}{|c|c|c|c|c|}
\hline $\begin{array}{l}\text { McCambridge et } \\
\text { al. } 2010 \text { [11] } \\
\text { Pre: Sept 2002- } \\
\text { Dec } 2003 \\
\text { Post: Oct 2004- } \\
\text { July } 2005\end{array}$ & $\begin{array}{l}\text { Academic community hospital; three ICUs } \\
\text { Closed model }{ }^{f} \\
\text { Active }\end{array}$ & $\begin{array}{l}\text { Tele-ICU team (intensivist and critical care } \\
\text { nurse) (1900-0700) admitted new patients } \\
\text { and responded to phone calls from ICU } \\
\text { nurses, computer-generated alerts, and } \\
\text { radiographic abnormalities } \\
\text { Rounds for all monitored patients q2 hours }\end{array}$ & Not described & $\begin{array}{l}\text { Vistacom Inc, Allentown PA } \\
\text { Health information technology bundle: } \\
\text { EMR with automatic alerts (iMDsoft, } \\
\text { Needham MA); CPOE, electronic MAR } \\
\text { and bar-coded medication } \\
\text { administration, PACS (GE Healthcare, } \\
\text { Fairfield CT) }\end{array}$ \\
\hline $\begin{array}{l}\text { Morrison et al. } \\
\text { 2010 [12] } \\
\text { Pre: Dec 2002-Mar } \\
\text { 2003 } \\
\text { Post 1: Dec 2004- } \\
\text { Mar 2004 } \\
\text { Post 2: July-Oct } \\
\text { 20049 }\end{array}$ & $\begin{array}{l}\text { One community teaching hospital (medical } \\
\text { ICU, surgical ICU, cardiac ICU) and one } \\
\text { community nonteaching hospital (medical- } \\
\text { surgical ICU) } \\
\text { Open model } \\
\text { Active }\end{array}$ & $\begin{array}{l}\text { Admitting physician responsible for care } \\
\text { plan and determined involvement of tele- } \\
\text { ICU (four categories from emergency care } \\
\text { only to no restrictions) } \\
\text { Tele-intensivist reviewed all patient data at } \\
\text { least q4 hours (q1 hour for sickest patients) } \\
\text { At teaching hospital, tele-intensivist } \\
\text { supervised and taught housestaff "real-time" }\end{array}$ & $\begin{array}{l}\text { Physician adoption of high-level } \\
\text { (unrestricted) tele-ICU care differed } \\
\text { (teaching hospital, 25\% of physicians [post } \\
\text { one], 57\% [post two]; nonteaching hospital, } \\
9 \% \text { [post one], } 27 \% \text { [post two]) }\end{array}$ & $\begin{array}{l}\text { VISICU Inc. (elCU CARE), Baltimore MD, } \\
\text { including "Sentry Alerts" software }\end{array}$ \\
\hline $\begin{array}{l}\text { Lilly et al. } 2011 \\
\text { [29] } \\
\text { Pre: April 2005- } \\
\text { Feb } 2007 \\
\text { Post: (staggered } \\
\text { roll-out) Aug } \\
\text { 2006-Sept } 2007\end{array}$ & $\begin{array}{l}\text { Academic medical center; seven ICUs: three } \\
\text { medical, three surgical, and one mixed } \\
\text { cardiovascular } \\
\text { Closed model } \\
\text { Active }\end{array}$ & $\begin{array}{l}\text { Tele-ICU (hospital staff intensivist, affiliate } \\
\text { practitioner, systems analyst, } \geq \text { one data } \\
\text { clerks), } 24 \text { hours/day } \\
\text { Tele-ICU monitored 5-minute timed median } \\
\text { vital sign values on electronic flow sheet; } \\
\text { reviewed care; audited best-practice } \\
\text { adherence real-time; reviewed night-time } \\
\text { admissions; monitored electronic alerts, } \\
\text { intervened when responses of bedside } \\
\text { clinicians to in-room alarms delayed }\end{array}$ & $\begin{array}{l}\text { Tele-ICU reviewed care plan for } 48 \% \text { of } \\
\text { after-hours admissions ( } 46 \% \text { reviewed by } \\
\text { other methods in pre period) } \\
23943 \text { tele-ICU initiated interventions for } \\
\text { physiologic instability that affected care } \\
\text { plan ( } 76 \% \text { "major") }\end{array}$ & $\begin{array}{l}\text { VISICU Inc. (elCU CARE), Baltimore MD; } \\
\text { APACHE (Cerner Healthcare Solutions, } \\
\text { Kansas City MO) } \\
\text { Criticalware (UMass) software package } \\
\text { to audit best practices (glycemic } \\
\text { control; prevention of DVT, CRBSI, VAP) } \\
\text { None }\end{array}$ \\
\hline $\begin{array}{l}\text { Willmitch et al. } \\
\text { 2012 [32] } \\
\text { Staggered roll-out: } \\
\text { Dec 2005-July } \\
2007 \\
\text { Pre: } 1 \text { year before } \\
\text { roll-out } \\
\text { Post 1: year } 1 \text { after } \\
\text { roll-out Post 2: } \\
\text { year } 2 \text { after roll- } \\
\text { out Post 3: year } 3 \\
\text { after roll-outh }\end{array}$ & $\begin{array}{l}\text { Five community hospitals with } 10 \text { ICUs } \\
\text { Closed model in largest hospital ( } 28 \% \text { of } \\
\text { ICU beds in the study); otherwise open } \\
\text { model }\left.\right|^{c} \\
\text { Active }\end{array}$ & $\begin{array}{l}\text { Tele-ICU, staffed by one intensivist, three } \\
\text { critical care nurses, and one secretary, } 24 \\
\text { hours/day }\end{array}$ & $\begin{array}{l}\text { All admitting and consulting physicians ( } n= \\
2,607 \text { ) indicated level of tele-ICU } \\
\text { intervention for their patients: } 1 \% \text { selected } \\
\text { level I (emergency care only), } 97 \% \text { level II } \\
\text { (best-practices adjustments), } 2 \% \text { level III (no } \\
\text { restrictions) }\end{array}$ & $\begin{array}{l}\text { Philips VISICU eCare Manager } \\
\text { (Admission, discharge and transfer } \\
\text { interfaces), Philips Smart Alerts, Philips } \\
\text { VISICU camera system (Philips, } \\
\text { Amsterdam, Netherlands) } \\
\text { None }\end{array}$ \\
\hline
\end{tabular}

CPOE, computerized physician order entry; CRBSI, catheter-related bloodstream infection; DVT, deep vein thrombosis; EMR, electronic medical record; ICU, intensive care unit; MAR, medication administration record; PACS, picture archiving and communications system; VAP, ventilator-associated pneumonia. ${ }^{a}$ Active system: continuous data monitoring with computer-generated alerts; high-intensity passive: continuous data

monitoring without computer-generated alerts; low-intensity passive: no continuous data monitoring. ${ }^{b} W$ used data from both pre (baseline) periods. 'Open model refers to low-intensity on-site daytime intensivist staffing in which patients may be cared for in the ICU without the man

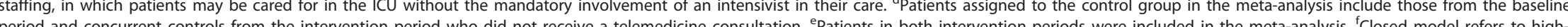
period and con

all post (intervention) periods. 
Figure 2, lower panel). Moderate statistical heterogeneity was found in both analyses $\left(I^{2}=70 \%\right.$ and $72 \%$, respectively).

Pooled data from seven studies (41,831 patients, 29,837 in the telemedicine group) showed a statistically significant difference in ICU length of stay (WMD, -0.62 days; $95 \% \mathrm{CI},-1.21$ to -0.04 days; $P=0.04$; Figure 3, upper panel). Similarly, a statistically significant difference was found in hospital length of stay (WMD, -1.26 days; $95 \% \mathrm{CI},-2.49$ to -0.03 days; $P=0.04$; Figure 3, lower panel) in six studies (40,613 patients, 29,197 in the telemedicine group). Both analyses showed high between-study heterogeneity $\left(I^{2}>90 \%\right)$.

\section{Subgroup analyses}

The effect of telemedicine on ICU mortality was similar in higher-quality studies (RR, $0.83 ; 95 \% \mathrm{CI}, 0.68$ to 1.02 ; $P=0.08$; six studies, 17,357 patients, 10,793 in the telemedicine group; Figure 4, upper panel) and lower-quality studies (RR, $0.69 ; 95 \% \mathrm{CI}, 0.40$ to $1.19 ; P=0.18$; three studies, 6,169 patients, 4,006 in the telemedicine group; Figure 4, lower panel). These RRs were not statistically different $(P=0.53$ for test for interaction).

Active or high-intensity passive telemedicine interventions (continuous data monitoring with or without computer-generated alerts) significantly decreased ICU mortality (RR, 0.78 ; 95\% CI, 0.64 to $0.95 ; P=0.01$; six studies, 21,384 patients, 13,911 in the telemedicine group; Figure 5, upper panel), compared with studies with passive interventions (remote intensivist consultation) (RR, 0.64; $95 \% \mathrm{CI}, 0.20$ to $2.07 ; P=0.45$; three studies, 2,142 patients, 888 in the telemedicine group; Figure 5, lower panel). However, these RRs were not statistically different ( $P=0.74$ for a test of interaction). When only active interventions were considered, no effect on ICU mortality was found (RR, 0.86; 95\% CI, 0.72 to $1.03 ; P=0.10$; four studies, 16,433 patients, 10,545 in the telemedicine group), and no difference from the effect in the passive group was found $(P=0.62$ for test of interaction).

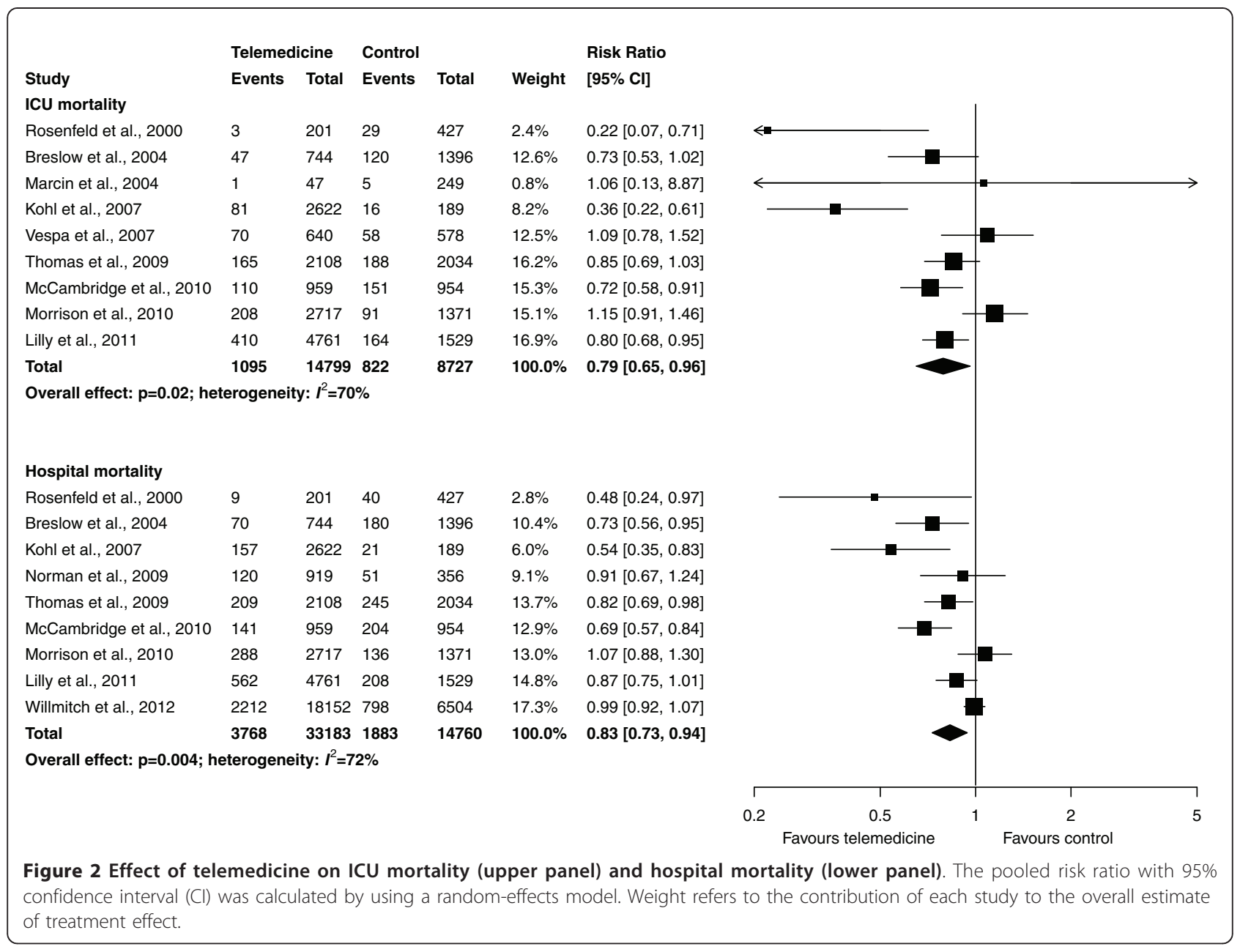




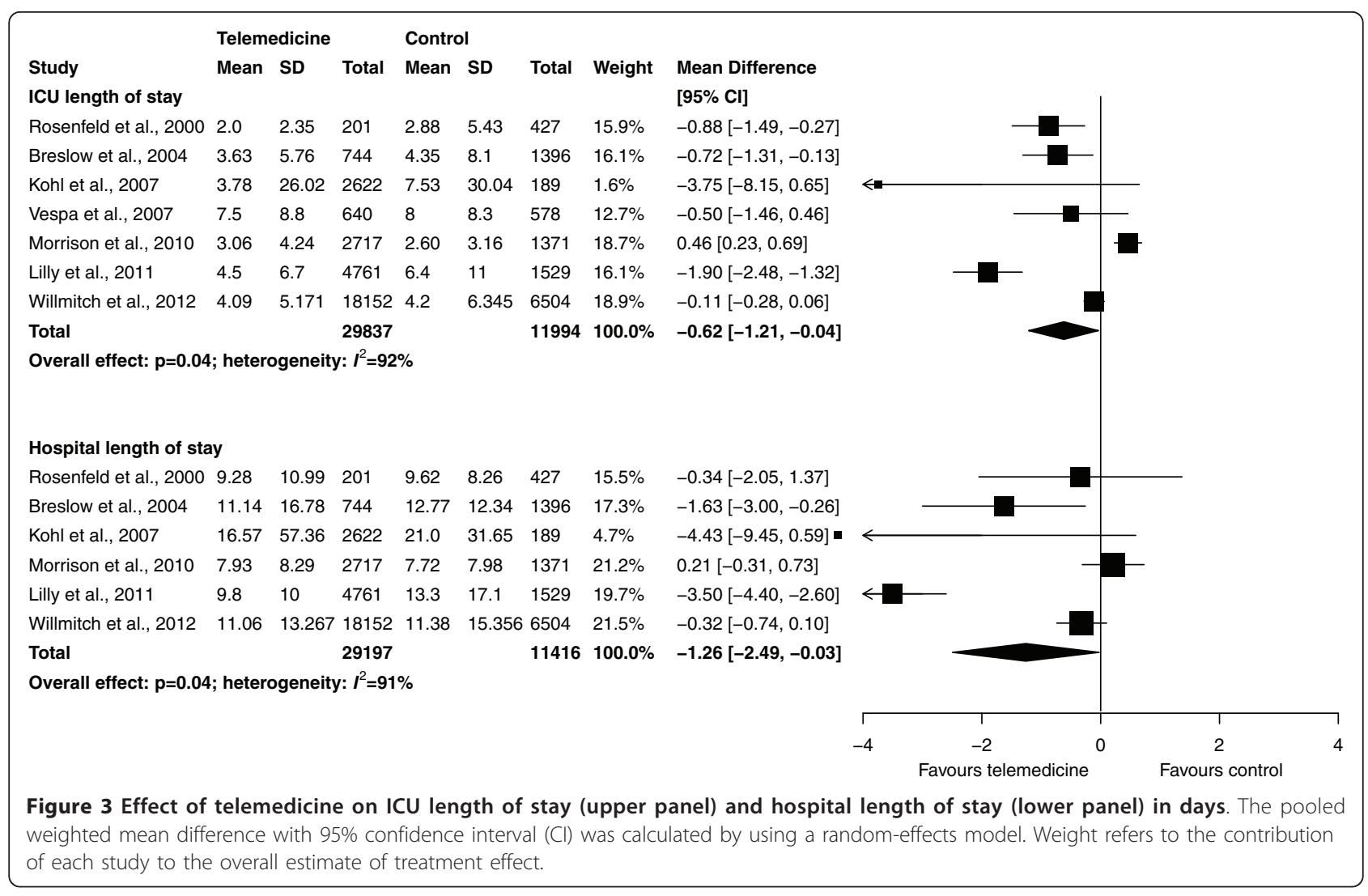

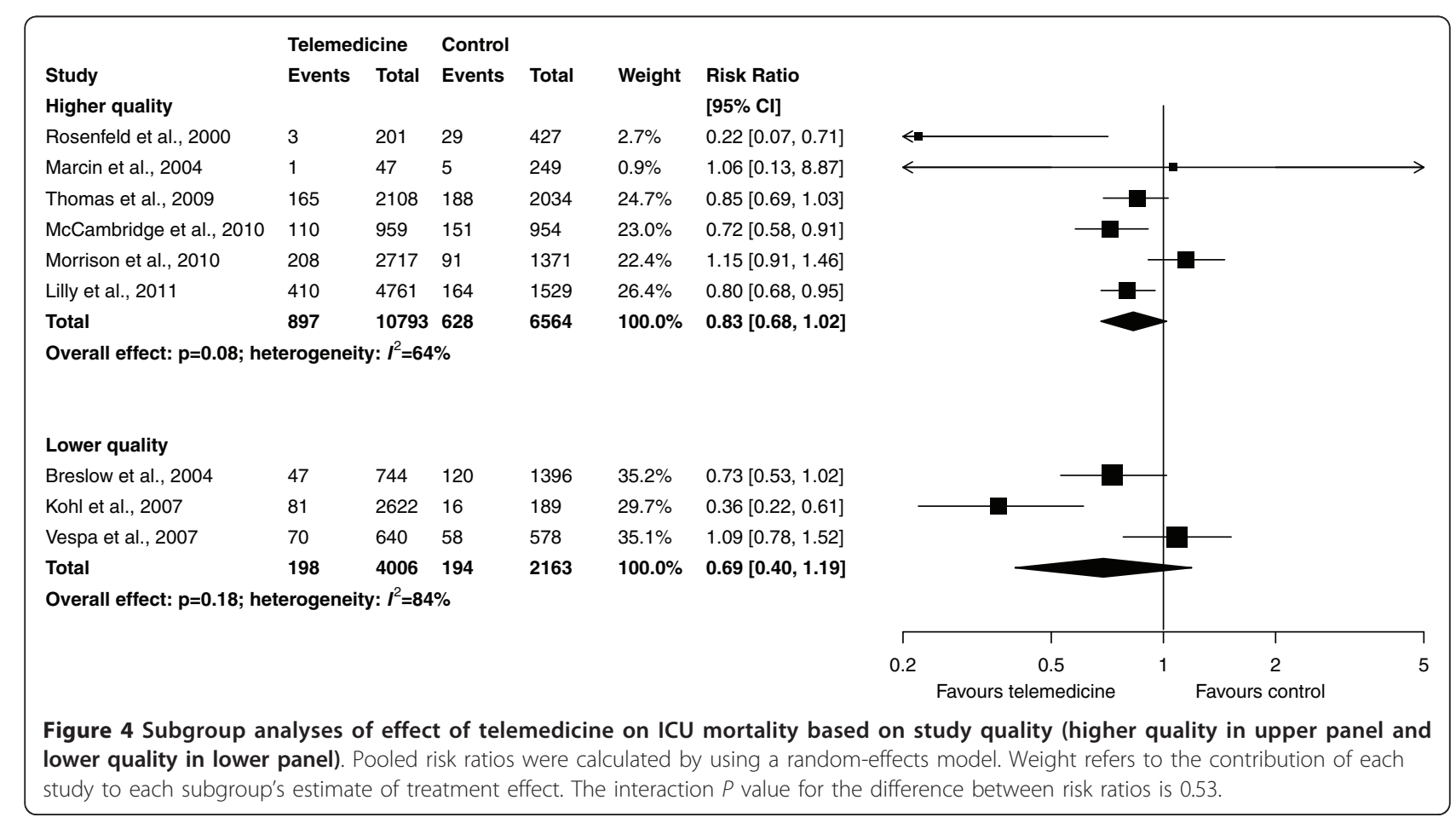




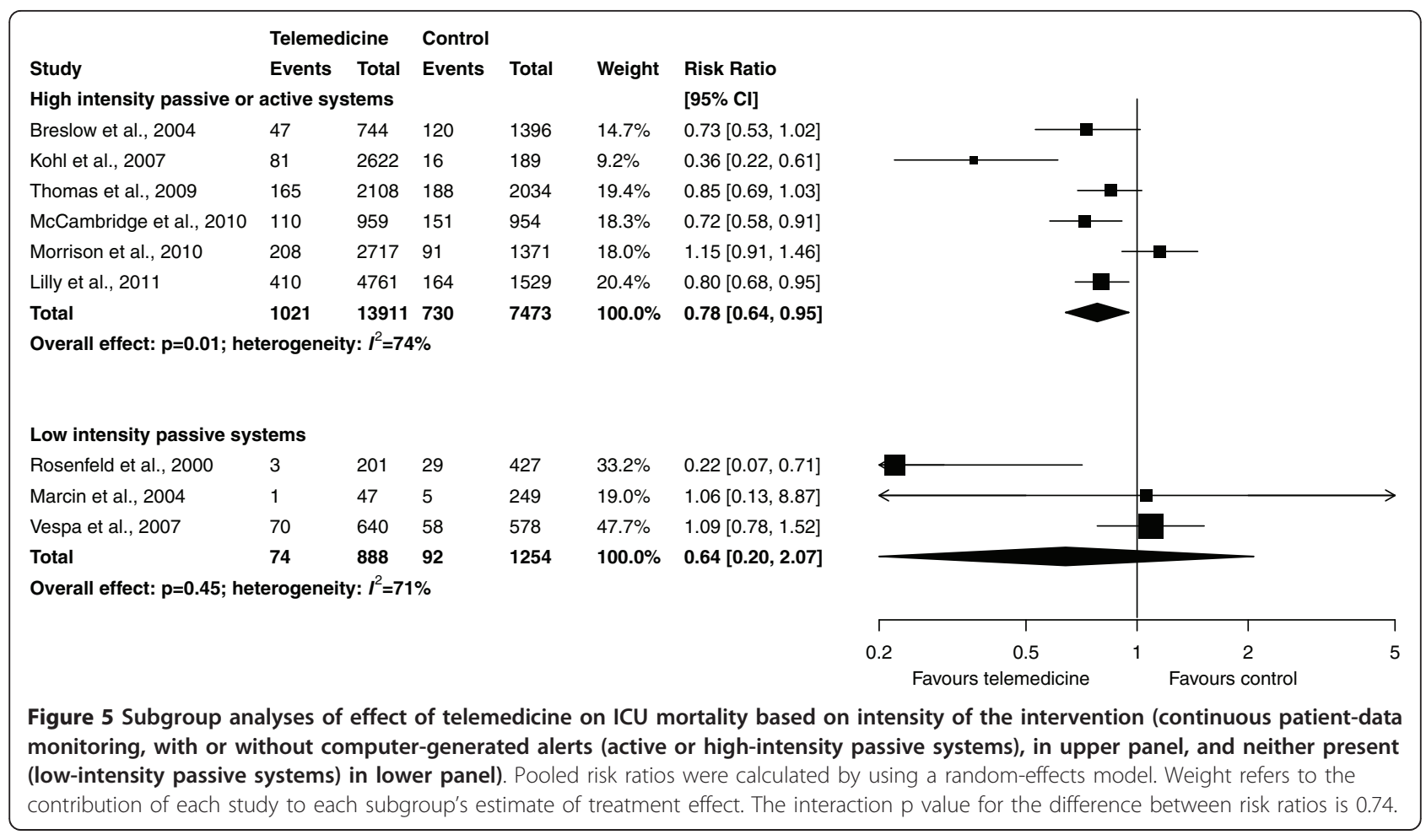

\section{Discussion}

In this systematic review and meta-analysis, we found 11 before/after observational studies, including 49,457 patients, that examined the effect of telemedicine on clinically important outcomes. Our main findings are that telemedicine reduced ICU and hospital mortality and lengths of stay in critically ill patients. Although the effect of telemedicine on ICU mortality was similar among active and passive systems, this subgroup analysis was underpowered to detect a true dose-response effect. Notwithstanding the methodologic limitations of observational studies and meta-analyses of unadjusted data, telemedicine appears to be a promising intervention to improve outcomes in the ICU and should be studied further.

Telemedicine systems may improve clinical outcomes by improving adherence to best practices in the ICU, which has been demonstrated to be poor [33]. One study [29] in this review found that telemedicine increased adherence to four best practices and reduced complications of ventilator-associated pneumonia and catheter-related bloodstream infections. Other studies not meeting inclusion criteria for this review have also shown improved adherence to best practices with telemedicine. For example, a recent cluster-randomized trial showed that a multifaceted knowledge-translation intervention that included education via telemedicine increased the adoption of targeted best practices in community hospitals with few resources dedicated to quality improvement [34]. Similarly, a before/ after study found a significant increase in the percentage of patients who received a daily sedative interruption with tele-ICU pharmacy support [35]. Last, one study reported improved nursing-staff satisfaction after the implementation of robotic tele-ICU [36]. However, with this exception [29], the studies in this review did not report process-ofcare measures sufficiently to address the hypothesis that lower mortality was associated with improved adherence to best practices.

Our results are similar to those of Young et al. [13], who found that telemedicine is associated with a reduction in ICU mortality (pooled odds ratio, 0.80; $95 \% \mathrm{CI}$, 0.66 to $0.97 ; P=0.02$ ), but not hospital mortality. Whereas Young et al. explored subgroup effects on the basis of vendor affiliation, our objective was to determine whether more technologically advanced telemedicine systems, with or without continuous patient-data monitoring, were associated with a statistically significant benefit on ICU mortality compared with less-advanced systems. Our subgroup analysis did not confirm a differential effect between these types of systems, even when the advanced-technology group was restricted to active systems with continuous patient-data monitoring and alerts. In addition to lack of statistical power in this analysis, the interventions used in included studies may have been misclassified because of the lack of a commonly adopted conceptual framework and vocabulary to describe components of the intervention, the intensity with which they were deployed, and the clinical actions attributable to their deployment [37]. 
Besides the lack of evidence to guide selection of telemedicine features important for clinical benefit, other issues should mitigate against widespread implementation. First, generalizability is limited, as studies were conducted in few hospitals in one country. Second, the impact of telemedicine likely depends on characteristics of the environment in which it is deployed, including ICU organization (such as physician-staffing model and use of protocols) and patient case mix. Studies generally reported limited relevant contextual details. Third, the costs of installation and maintenance [38], potential for malfunction and downtime, and impact of redeployment of intensivists away from bedside clinical care during labor shortages have not been analyzed. Fourth, telemedicine has been primarily examined in before/after observational studies, from which conclusions regarding causality may be confounded by secular trends in case-mix and other interventions. These issues, although shared by healthcare technology in general [39], imply that universal adoption of telemedicine should be depend on the results of future studies, the design of which should be informed by a robust understanding of system design and organizational factors associated with patient benefit.

Strengths of this review include several methods to minimize bias, including a comprehensive literature search, duplicate outcomes abstraction, consideration of important clinical outcomes, and use of an established method to assess study quality specific to nonrandomized studies [22]. Our review also has weaknesses. In the absence of any randomized trials of telemedicine, we included observational studies, which tend to overestimate the effects of an intervention [40] even with standard methods to adjust for differences between groups [40-42]. Our meta-analyses used unadjusted data and may have further exaggerated treatment effects. Although we believed the interventions were sufficiently similar in concept and execution to permit statistical aggregation, major differences occur in the components of this intervention, methods of deployment, and rates of adoption in the included studies. Moderate statistical heterogeneity was seen in our primary outcome that persisted in subgroups defined by study quality and telemedicine technology. Therefore, even among studies using advanced telemedicine interventions, our review cannot identify with certainty the components essential to success. Last, our subgroup analyses had few studies; for example, the lower-technology (low-intensity passive) telemedicine subgroup had only three studies. The power to detect clinically important subgroup effects was therefore limited.

\section{Conclusions}

Telemedicine is a promising technology to reduce mortality in the critically ill. Recently, the Critical Care
Societies Collaborative proposed a comprehensive research agenda in ICU telemedicine, including development of a conceptual framework to describe the telemedicine system and the recipient ICUs and elucidation of mechanisms of telemedicine's effects on downstream clinical outcomes by analyzing effects on structure and process-of-care variables [37]. We believe that this complex intervention, similar to others in the ICU, warrants eventual evaluation in a cluster-randomized trial. Important areas of research that would inform the design of such a trial, in addition to those described, include individual patient meta-analysis of existing studies to permit adjustment for hospital and patient-level characteristics to identify patients and centers most likely to benefit, assessment of clinical equipoise (physician and nursing attitudes to ICU telemedicine) by using qualitative methods [43], and pilot observational studies to establish the optimal telemedicine technology configuration and dose tailored to ICU organization and case mix.

\section{Key messages}

- We found 11 before/after observational studies including 49,457 patients that examined the effect of telemedicine on clinically important outcomes.

- Pooled unadjusted data from nine studies showed that telemedicine reduced ICU mortality (RR, 0.79; 95\% CI, 0.65 to $0.96 ; P=0.02$ ) and hospital mortality (RR, 0.83 ; $95 \% \mathrm{CI}, 0.73$ to $0.94 ; P=0.004$ ); reductions in ICU and hospital lengths of stay were also statistically significant.

- The effect of telemedicine on ICU mortality was similar in active or high-intensity passive systems (continuous patient-data monitoring with or without electronic alerts) compared with low-intensity passive systems (remote intensivist consultation only), but this subgroup analysis was underpowered.

- Future research should establish the optimal telemedicine technology configuration and dose tailored to ICU organization and case mix.

\section{Additional material} Additional file 1: Supplementary details of method and results. This
file contains details of the search strategy (Appendix 1), summary of
reasons for excluding 62 studies (Appendix B), assessment of
methodologic quality of the included studies (Appendix C), and a funnel
plot for the meta-analysis of ICU mortality (Appendix D).

\section{Abbreviations}

$\mathrm{Cl}$ : confidence interval; ICU: intensive care unit; LOS: length of stay; RR: risk ratio; WMD: weighted mean difference.

\section{Acknowledgements}

We appreciate the thoughtful advice of Andrew Hutchings and Hannah Wood in the preparation of this manuscript and the assistance of Ruxandra 
Pinto in preparation of the Figures 2 through 5. This study received no funding. Preliminary results have been presented in abstract form [44].

\section{Author details}

'Department of Medicine, Toronto Western Hospital, and University of Toronto, McLaughlin Wing 2-411H, 399 Bathurst Street, Toronto ON M5T 2S8, Canada. ${ }^{2}$ Department of Critical Care Medicine and Sunnybrook Research Institute, Sunnybrook Health Sciences Centre and University of Toronto, 2075 Bayview Avenue Room D1.08, Toronto ON M4N 3M5, Canada.

\section{Authors' contributions}

MEW conceived of the study, searched the literature, selected studies for inclusion, abstracted data, analyzed data, wrote the first draft of the manuscript, and revised the manuscript. NKJA selected studies for inclusion, abstracted data, analyzed data, and revised the manuscript. Both authors approved the final manuscript.

\section{Competing interests}

The authors declare that they have no competing interests.

Received: 31 January 2012 Revised: 8 June 2012

Accepted: 18 July 2012 Published: 18 July 2012

\section{References}

1. Rubenfeld GD, Angus DC: Are intensivists safe? Ann Intern Med 2008, 148:877-879.

2. Kahn JM, Brake $H$, Steinberg KP: Intensivist physician staffing and the process of care in academic medical centres. Qual Saf Health Care 2007, 16:329-333.

3. Pronovost PJ, Angus DC, Dorman T, Robinson KA, Dremsizov T, Young $T L$ Physician staffing patterns and clinical outcomes in critically ill patients: a systematic review. JAMA 2002, 288:2151-2162

4. Pronovost PJ, Waters $\mathrm{H}$, Dorman $\mathrm{T}$ : Impact of critical care physician workforce for intensive care unit physician staffing. Curr Opin Crit Care 2001, 7:456-459.

5. Angus DC, Kelley MA, Schmitz RJ, White A, Popovich J Jr, (COMPACCS): Caring for the critically ill patient: current and projected workforce requirements for care of the critically ill and patients with pulmonary disease: can we meet the requirements of an aging population? JAMA 2000, 284:2762-2770.

6. Cummings J, Krsek C, Vermoch K, Matuszewski K, for University Health System Consortium task force: Intensive care unit telemedicine: review and consensus recommendations. Am J Med Qual 2007, 22:239-250.

7. Nguyen $Y$-L, Kahn JM, Angus DC: Reorganizing adult critical care delivery: the role of regionalization, telemedicine, and community outreach. Am J Respir Crit Care Med 2010, 181:1164-1169.

8. Breslow MJ, Rosenfeld BA, Doerfler M, Burke G, Yates G, Stone DJ, Tomaszewicz P, Hochman R, Plocher DW: Effect of a multiple-site intensive care unit telemedicine program on clinical and economic outcomes: an alternative paradigm for intensivist staffing. [Erratum appears in Crit Care Med 2004, 32:1632]. Crit Care Med 2004, 32:31-38.

9. Thomas EJ, Lucke JF, Wueste L, Weavind L, Patel B: Association of telemedicine for remote monitoring of intensive care patients with mortality, complications, and length of stay. JAMA 2009, 302:2671-2678.

10. Rosenfeld BA, Dorman T, Breslow MJ, Pronovost P, Jenckes M, Zhang N, Anderson $\mathrm{G}$, Rubin $\mathrm{H}$ : Intensive care unit telemedicine: alternate paradigm for providing continuous intensivist care. Crit Care Med 2000, 28:3925-3931.

11. McCambridge M, Jones K, Paxton H, Baker K, Sussman EJ, Etchason J: Association of health information technology and teleintensivist coverage with decreased mortality and ventilator use in critically ill patients. Arch Intern Med 2010, 170:648-653.

12. Morrison JL, Cai Q, Davis N, Yan Y, Berbaum ML, Ries M, Solomon G: Clinical and economic outcomes of the electronic intensive care unit: results from two community hospitals. Crit Care Med 2010, 38:2-8.

13. Young LB, Chan PS, Lu X, Nallamothu BK, Sasson C, Cram PM: Impact of telemedicine intensive care unit coverage on patient outcomes: a systematic review and meta-analysis. Arch Intern Med 2011, 171:498-506

14. Altman DG: Practical Statistics for Medical Research London: Chapman and Hall; 1991
15. Chen J, Fang XL, Fang Q, Cai HL, Su Q, Zhang YT: The clinical application of remote critical care network. Zhongguo Wei Zhong Bing Ji Jiu Yi Xue 2009, 21:679-681.

16. Dimand RJ, Marcin JP, Kallas HJ, Struve SN: Use of telemedicine to provide pediatric critical care inpatient consultations to underserved rural Northern California. Crit Care Med 2001, 29:A112.

17. Rogove H, Atkins C, Kramer J, Vespa PM, Miller C, Botticella M, Chalfin D: Enhanced access to neurointensivists through a telemedicine program [abstract]. Crit Care Med 2009, 37:A2.

18. Turner G, Melby K: Predictable changes in medical-surgical ICU patient hospital mortality with evolving intensivist services and evolving then devolving telemedicine unit services [abstract]. Crit Care Med 2009, 37: A130.

19. Latif A, Romig M: A consultative telemedicine service improves compliance with best practice guidelines in a highly staffed intensive care unit [abstract]. Crit Care Med 2010, 38:A582.

20. Marini CP, Sharma C, Barrera R, McNelis J, Ritter G, Ravikumar TS: The effect of telerounding in a surgical intensive care unit [abstract]. Crit Care Med 2007, 35:A627.

21. Zawada ET Jr, Herr P, Larson D, Fromm R, Kapaska D, Erickson D: Impact of an intensive care unit telemedicine program on a rural health care system. Postgrad Med 2009, 121:160-170.

22. Wells GA, Shea B, O'Connell D, Peterson J, Welch V, Losos M, Tugwell P. The Newcastle-Ottawa Scale (NOS) for assessing the quality of nonrandomised studies in meta-analyses.[http://www.ohri.ca/programs/ clinical_epidemiology/oxford.asp].

23. Higgins JP, Thompson SG: Quantifying heterogeneity in a meta-analysis. Stat Med 2002, 21:1539-1558.

24. Higgins JP, Thompson SG, Deeks JJ, Altman DG: Measuring inconsistency in meta-analyses. BMJ 2003, 327:557-560

25. Peters $J$, Sutton AJ, Jones DR, Abrams KR, Rushton L: Comparison of two methods to detect publication bias in meta-analysis. JAMA 2006, 295:676-680.

26. Fleiss JL: The statistical basis of meta-analysis. Stat Methods Med Res 1993, 2:121-145

27. Marcin JP, Nesbitt TS, Kallas HJ, Struve SN, Traugott CA, Dimand RJ: Use of telemedicine to provide pediatric critical care inpatient consultations to underserved rural Northern California. J Pediatr 2004, 144:375-380.

28. Vespa PM, Miller C, Hu X, Nenov V, Buxey F, Martin NA: Intensive care unit robotic telepresence facilitates rapid physician response to unstable patients and decreased cost in neurointensive care. Surg Neurol 2007 67:331-337.

29. Lilly CM, Cody S, Zhao H, Landry K, Baker SP, Mcllwaine J, Chandler MW, Irwin RS, University of Massachusetts Memorial Critical Care Operations Group: Hospital mortality, length of stay, and preventable complications among critically ill patients before and after tele-ICU reengineering of critical care processes. JAMA 2011, 305:2175-2183.

30. Kohl BA, Gutsche JT, Kim P, Sites FD, Ochroch EA: Effect of telemedicine on mortality and length of stay in a University hospital [abstract]. Crit Care Med 2007, 35:A111.

31. Norman V, French R, Hassan E, Kabani N, Mizell P, Stone DJ, Griebel J Jr, Tragico E: Effect of a telemedicine facilitated program on intensive care unit length of stay (LOS) and financial performance [abstract]. Crit Care Med 2009, 37:A3.

32. Willmitch B, Golembeski S, Kim SS, Nelson LD, Gidel L: Clinical outcomes after telemedicine intensive care unit implementation. Crit Care Med 2012, 40:450-454.

33. Lilly CM, Zuckerman $\boxplus$, Badawi O, Riker RR: Benchmark data from more than 240,000 adults that reflect the current practice of critical care in the United States. Chest 2011, 140:1232-1242.

34. Scales DC, Dainty K, Pinto R, Fowler RA, Adhikari NK, Zwarenstein M: A multifaceted intervention for quality improvement in a network of intensive care units: a cluster randomized trial. JAMA 2011, 305:363-372.

35. Forni A, Skehan N, Hartman CA, Yogaratnam D, Njoroge M, Schifferdecker C, Lilly CM: Evaluation of the impact of a tele-ICU pharmacist on the management of sedation in critically ill mechanically ventilated patients. Ann Pharmacother 2010, 44:432-438.

36. Rincon F, Vibbert M, Childs V, Fry R, Caliguri D, Urtecho J, Rosenwasser R, Jallo J: Implementation of a model of robotic tele-presence (RTP) in the Neuro-ICU: effect on critical care nursing team satisfaction. Neurocrit Care 2012. 
37. Kahn JM, Hill NS, Lilly CM, Angus DC, Jacobi J, Rubenfeld GD, Rothschild JM, Sales AE, Scales DC, Mathers JA: The research agenda in ICU telemedicine: a statement from the Critical Care Societies Collaborative. Chest 2011, 140:230-238.

38. Franzini L, Thomas E: Costs and effectiveness of tele-ICUs in reducing morbidity and mortality in intensive care units. J Med Econ 2008, 11:165-169.

39. Chaudhry B, Wang J, Wu S, Maglione M, Mojica W, Roth E, Morton SC, Shekelle PG: Systematic review: impact on health information technology on quality, efficiency, and costs of medical care. Ann Intern Med 2006, 144:742-752.

40. Deeks JJ, Dinnes J, D'Amico R, Sowden AJ, Sakarovitch C, Song F, Petticrew M, Altman DG, International Stroke Trial Collaborative Group, European Carotid Surgery Trial Collaborative Group: Evaluating nonrandomised intervention studies. Health Technol Assess 2003, 7:1-173.

41. Golder S, Loke Y, McIntosh HM: Room for improvement? A survey of methods used in systematic reviews of adverse effects. BMC Med Res Methodol 2006, 6:1-6.

42. Audrige L, Bhandari M, Griffin D, Middleton P, Reeves BC: Systematic reviews of nonrandomised clinical studies in the orthopaedic literature. Clin Orthop Rel Res 2004, 427:249-257.

43. Young LB, Chan PS, Cram P: Staff acceptance of tele-ICU coverage: a systematic review. Chest 2011, 139:279-288.

44. Wilcox ME, Adhikari NK: Effect of telemedicine on mortality in critically ill patients: systematic review and meta-analysis. Am J Respir Crit Care Med 2012, 185:A2867.

\section{doi:10.1186/cc11429}

Cite this article as: Wilcox and Adhikari: The effect of telemedicine in critically ill patients: systematic review and meta-analysis. Critical Care 2012 16:R127.

\section{Submit your next manuscript to BioMed Central and take full advantage of:}

- Convenient online submission

- Thorough peer review

- No space constraints or color figure charges

- Immediate publication on acceptance

- Inclusion in PubMed, CAS, Scopus and Google Scholar

- Research which is freely available for redistribution

Submit your manuscript at www.biomedcentral.com/submit 